Pacific Journal of Mathematics

THE WEAK ENVELOPE OF HOLOMORPHY FOR ALGEBRAS 


\title{
THE WEAK ENVELOPE OF HOLOMORPHY FOR ALGEBRAS OF HOLOMORPHIC FUNCTIONS
}

\author{
ALAN T. HUCKLEBERRY
}

The object of this paper is to study analytic continuation of algebras of functions holomorphic on complex spaces of dimension greater than 1. Classically this has been done by putting complex structure on the maximal spectrum of the algebra so that the spectrum is a Stein space with respect to the induced algebra of holomorphic functions. Grauert has given non-pathological examples where this is not possible. In the present paper the axioms of a Stein space have been weakened and the weak envelope of holomorphy has been constructed for a certain type of algebra. In particular, if the algebra $A$ separates points and gives local coordinates on a complex space $X$ then the weak envelope of holomorphy for the pair, $(X, A)$ is obtained.

1. Introduction. In this paper a complex space, unless otherwise stated, will mean a normal, connected, reduced complex space. We will let $H(X)$ denote the algebra of functions holomorphic on a complex space $X$. A complex space $E$ is said to be the envelope of holomorphy of a complex space $X$ if the following conditions are satisfied:

(1) There is a holomorphic mapping $\tau: X \rightarrow E$ such that $\tau(X)$ is open in $E$.

(2) The map $\tau^{*}: H(E) \rightarrow H(X)$ is an algebra isomorphism.

(3) The complex space $E$ is a Stein space.

It is known that if $X$ has an envelope of holomorphy, $E$, and $\tau^{\prime}: X \rightarrow E^{\prime}$ satisfies (1) and (2) above then there is a biholomorphic mapping $\varphi: E^{\prime} \rightarrow E$ such that $\tau=\varphi \circ \tau^{\prime}$. Moreover, the spectrum of $H(X)$, $S(H(X))$, has the structure of a complex space such that it is biholomorphically equivalent to $E$ in a natural way [2].

Grauert has provided an example of a complex manifold $X$ with $H(X)$ containing local coordinates and separating points, but $S(H(X))$ contains a point no neighborhood of which has the structure of an analytic variety [1]. Thus, in order to investigate the envelope of holomorphy problem, it makes sense to either modify the notion of a complex space, as Grauert has suggested [1], or to weaken the constraints on an envelope of holomorphy. In this paper we have taken the latter route. We have weakened the restrictions (1), (2), and (3) above, while preserving the maximality property. Our results, which apply to a class of algebras which includes many algebras which are not of the form $H(X)$, can be described as follows. 
Let $A$ be an algebra of functions holomorphic on a complex space $X$. Let $A^{*}$ be the quotient field of $A$ and

$S_{A}=\left\{\left(x_{1}, x_{2}\right) \in X \times X \mid f\left(x_{1}\right)=f\left(x_{2}\right) \forall f \in A^{*}\right.$ such that $f$ is holomorphic at $x_{1}$ and $\left.x_{2}\right\}$.

Definition 1.1. Let $A$ be an algebra of functions holomorphic on a complex space $X$. We say that $A$ weakly identifies the points $x_{1}$ and $x_{2}$ in $X$ if

1. Every $f \in A^{*}$ which is holomorphic at $x_{1}$ is also holomorphic at $x_{2}$ and vice versa, and $f\left(x_{1}\right)=f\left(x_{2}\right)$ for all such holomorphic quotients and 2. The dimension of $S_{A}$ at $\left(x_{1}, x_{2}\right)$ is at least the dimension of $X$.

The algebra $A$ is said to weakly separate $x_{1}$ from $x_{2}$ if it does not weakly identify $x_{1}$ and $x_{2}$. Finally, we say that $A$ weakly separates points on $X$ if every two points from $X$ are weakly separated by $A$.

Definition 1.2. Let $A$ be an algebra of functions holomorphic on an $n$-dimensional complex space $X$. We say that $A$ covers a variety at $p \in X$ if there is an open neighborhood $W$ of $p$ and a map $F: W \rightarrow C^{m}$ onto an $n$-dimensional subvariety of an open set in $C^{m}$ such that $F=\left(f_{1}, \cdots, f_{m}\right)$ with $f_{i} \in A$ and $F$ is an open mapping onto its image.

We now are able to describe the type of complex space which is our candidate for the weak envelope of holomorphy.

Definition 1.3. Let $A$ be an algebra of functions holomorphic on a complex space $X$. We say that $X$ is weakly Stein with respect to $A$ if

( $\alpha$ ) The algebra $A$ weakly separates points on $X$.

( $\beta$ ) The algebra $A$ covers a variety at every point of $X$.

$(\gamma)$ For every $p \in X$ the quotient field $A^{*}$ contains functions holomorphic at $p$ which give coordinates for $X$ in a neighborhood of $p$

and

( $\delta$ ) If there is a complex space $X^{\prime}$ containing $X$ and an algebra $A^{\prime} \subseteq H\left(X^{\prime}\right)$ satisfying $(\alpha),(\beta)$, and $(\gamma)$ such that the restriction map $r: A^{\prime} \rightarrow A$ is an isomorphism then $X^{\prime}=X$.

Let $\mathcal{O}_{p}$ be the stalk of the structure sheaf on $X$ at $p$. If $A \subset H(X)$ is an algebra then we define $h_{x}: A \rightarrow \mathcal{O}_{x}$ as the restriction map. We will prove a theorem which clarifies the meaning of weak separation of points. In the above notation this theorem can be stated as follows: 
TheOREM 1. Suppose $A^{*}$ contains coordinates at $x_{1}, x_{2} \in X$. Then $A$ weakly separates $x_{1}$ from $x_{2}$ if and only if $h_{x_{1}} \circ h_{x_{2}}^{-1}$ can not be extended to a continuous isomorphism of $\mathcal{O}_{x_{2}}$ onto $\mathcal{O}_{x_{1}}$.

We now wish to define a class of algebras for which the weak envelope of holomorphy exists.

Definition 1.4. An algebra $A$ of functions holomorphic on a complex space $X$ is said to be ample on $X$ if for every $p \in X$ the following two conditions hold:

(a) The algebra $A$ covers a variety at $p$.

(b) There is a neighborhood $W$ of $p$, a holomorphic mapping $\varphi$ of $W$ onto a complex space $V$, an algebra $\hat{A} \subset H(V)$ such that $\varphi^{*}: \hat{A} \rightarrow A$ is an algebra isomorphism and $\hat{A}^{*}$ contains local coordinates at each point of $V$.

We will prove the following theorem concerning abstract algebras.

THEOREM 2. Let $A$ be an algebra over $C$. Then there is a not necessarily connected) complex space $\operatorname{Rep} A$ called the representation space of $A$, and an algebra $\hat{A} \subset H(\operatorname{Rep} A)$ such that each connectivity component of $\operatorname{Rep} A$ is weakly Stein with respect to $\hat{A}$. Furthermore, there is an algebra homomorphism $\hat{h}$ of $A$ onto $\hat{A}$ such that for any other algebra homomorphism $h^{\prime}$ of $A$ onto $A^{\prime}$ an ample algebra on $a$ complex space $X^{\prime}$ there is a unique holomorphic mapping $\varphi$ of $X^{\prime}$ onto an open subset of $\operatorname{Rep} A$ such that for every $f \in A, h^{\prime}(f)=\hat{h}(f) \circ \varphi$. The mapping $\varphi$ is 1-1 if and only if $A^{\prime}$ weakly separates points on $X^{\prime}$.

As a corollary to the above theorem we obtain the weak envelope of holomorphy for an ample algebra of holomorphic functions:

THEOREM 3. Let $A$ be an ample algebra of functions holomorphic on a complex space $X$. Then there is a complex space $E$ and an algebra $\hat{A} \subset H(E)$ such that

(1) The space $E$ is weakly Stein with respect to $\hat{A}$.

(2) There is a holomorphic mapping $\tau$ of $X$ onto an open subset of $E$ such that $\tau^{*}: \hat{A} \rightarrow A$ is an algebra isomorphism.

(3) If $\tau^{\prime}$ is a holomorphic mapping of $X$ onto an open subset of a complex space $X^{\prime}$ and there is an ample algebra $A^{\prime} \subset H\left(X^{\prime}\right)$ such that $\tau^{\prime *}: A^{\prime} \rightarrow A$ is an isomorphism then there is a unique holomorphic mapping $\varphi$ of $X^{\prime}$ onto an open subset of $E$ such that for every $f \in A$, 
$\left(\tau^{*}\right)^{-1} \circ \varphi=\left(\tau^{* *}\right)^{-1}(f)$. The map $\varphi$ is 1-1 if and only if $A^{\prime}$ is weakly separating on $X^{\prime}$.

The pair $(E, \hat{A})$ in Theorem 3 is unique up to biholomorphic equivalence and is called the weak envelope of holomorphy for the pair $(X, A)$. We note that Theorem 1 says that $\tau$ identifies $x_{1}$ and $x_{2}$ if and only if $A$ "looks exactly the same at $x_{1}$ as it does at $x_{2}$ ". This, coupled with the maximality property contained in Theorem 3, shows that $E$ is the complex space where $A$ "lives".

The theory presented here answers a question posed to me by Royden and generalizes to higher dimensions his Riemann surface representative space [5]. The germs of this theory and some results in the 2-dimensional case can be found in [3]. I wish to thank Professor Royden for numerous helpful conversations.

2. Separation of points. A family of functions $\mathscr{F}$ on a topological space $X$ separates points on $X$ if for $p, q \in X$ there exists $f \in \mathscr{F}$ such that $f(p) \neq f(q)$. If one takes the algebra $H(X)$ of holomorphic functions on a complex space $X$ and considers the induced algebra $\hat{H}$ on $S(H(X))$ then he finds that $\hat{H}$ separates points on $S(H(X))$. In a sense this is one reason that $S(H(X))$ may not have complex structure, as the level sets of $H(X)$ must be collapsed to points. Thus, our first step toward obtaining a weak envelope of holomorphy which is a complex space is to weaken the definition of separation of points.

In all that follows $A$ is an algebra of functions holomorphic on a complex space $X$ and $A^{*}$ is its quotient field.

\section{LEMMA 2.1. Let}

$$
\begin{aligned}
S_{A}= & \left\{\left(x_{1}, x_{2}\right) \in X \times X \mid f\left(x_{1}\right)=f\left(x_{2}\right) \forall f \in A^{*} \text { such that } f\right. \\
& \text { is holomorphic at } \left.x_{1} \text { and } x_{2}\right\} .
\end{aligned}
$$

Then $S_{A}$ is an analytic subvariety of $X \times X$.

Proof. For every $f \in A^{*}$ we have the meromorphic correspondence $X \times X \stackrel{(f, f)}{\circ} P_{1} \times P_{1}$ which has graph $G_{(f, f)}$ such that

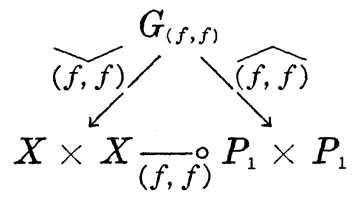

is commutative, $(\widehat{f, f})$ is proper holomorphic and $(\widehat{f, f})$ is holomorphic [6]. Let $\Delta$ be the diagonal of $P_{1} \times P_{1}$. Then $(f, f)^{-1}[\Delta]=V$ is an 
analytic subvariety of $G_{(f, f)}$ and, since $(\widetilde{f, f})$ is proper, $(\widetilde{f, f})[V]$ is an analytic subvariety of $X \times X$. Let $L(f)=(\hat{f, f})[V]$. Now $S_{A}=$ $\bigcap_{f \in A^{*}} L(f)$. Since each $L(f)$ is an analytic subvariety of $X \times X, S_{A}$ is an analytic subvariety of $X \times X$.

Thus we may speak of the dimension of $S_{A}$ at a given point and to use it in the definition of weak separation of points, Definition 1.1. We attempt to clarify the meaning of weak separation with the following theorem.

THEOREM 1. Let $A$ be an algebra of functions holomorphic on a complex space $X$. Let $x_{1}, x_{2} \in X$ and suppose $A^{*}$ contains coordinates at $x_{1}$ and $x_{2}$. Let $h_{x_{i}}: A \rightarrow \mathcal{O}_{x_{i}}$ be the restriction maps for $i=1,2$. Then $A$ weakly separates $x_{1}$ from $x_{2}$ if and only if $h_{x_{1}} \circ h_{x_{2}}^{-1}$ can not be extended to a continuous algebra isomorphism of $\mathcal{O}_{x_{2}}$ onto $\mathcal{O}_{x_{1}}$.

Proof. Suppose $h_{x_{1}} \circ h_{x_{2}}^{-1}$ extends to a continuous isomorphism of $\mathcal{O}_{x_{2}}$ onto $\mathcal{O}_{x_{1}}, h$. Let $f, g \in h_{x_{1}}[A]$ and suppose $q=f / g \in \mathcal{O}_{x_{1}}$. Then $h(q) \in \mathcal{O}_{x_{2}}$ and $h(q)=h(f) / h(g)$. Furthermore, since $h(1)=1$ and units are mapped into units, $q\left(x_{1}\right)=q\left(x_{2}\right)$. Suppose $q_{1}^{i}, \cdots, q_{n_{i}}^{i}$ give coordinates at $x_{i}$, where $q_{j}^{i} \in A^{*}$ and $i=1,2$. By the above remarks, each $q_{j}^{i}$ is holomorphic at both $x_{1}$ and $x_{2}$. Let $Q=\left(q_{1}^{1}, \cdots, q_{n_{1}}^{1}, q_{2}^{2}, \cdots, q_{n_{2}}^{2}\right)$ and $V_{1}$ (resp. $V_{2}$ ) be a neighborhood of $x_{1}$ (resp. $x_{2}$ ) such that $Q_{i}=$ $\left.Q\right|_{V_{i}}: V_{i} \rightarrow W_{i}$ is biholomorphic for $i=1,2$.

Now $W_{1} \cap W_{2}$ is a subvariety of an open set in $\boldsymbol{C}^{n_{1}+n_{2}}$ which contains $Q_{1}\left(x_{1}\right)=Q_{2}\left(x_{2}\right)$. Suppose $\operatorname{dim}_{Q_{1}\left(x_{1}\right)} W_{1} \cap W_{2} \leqq \operatorname{dim} X-1$. Then there is a function $f$ holomorphic in a neighborhood of $Q_{1}\left(x_{1}\right)$ in $C^{n_{1}+n_{2}}$ such that $f \circ Q_{1} \equiv 0$ and $f \circ Q_{2} \neq 0$. Since $f$ is given by a convergent

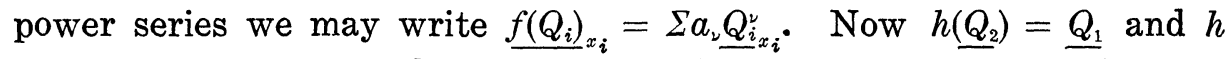
is a continuous isomorphism. Thus $h\left(\underline{f\left(Q_{2}\right)_{x_{2}}}\right)=f\left(Q_{1}\right)_{x_{1}}$. But $\underline{f\left(Q_{1}\right)_{x_{1}}=0}$

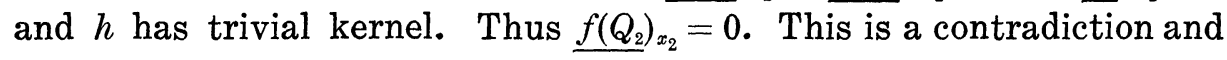
therefore $\operatorname{dim}_{Q\left(x_{1}\right)} W_{1} \cap W_{2}=\operatorname{dim} X=n$.

If $\operatorname{dim}_{\left(x_{1}, x_{2}\right)} S_{A} \leqq n-1$ then there must be $q \in A^{*}$ which is holomorphic at $x_{1}$ and $x_{2}$ such that if we replace $Q$ above by $(Q, q)$ then $\operatorname{dim}_{Q\left(x_{1}\right)} W_{1} \cap W_{2} \leqq n-1$. By the same argument as above we reach a contradiction. Hence $\operatorname{dim}_{\left(x_{1}, x_{2}\right)} S_{A} \geqq n$. Since we have already shown that the first criterion for weak identification is satisfied, $x_{1}$ and $x_{2}$ are weakly identified by $A$.

Conversely, suppose $x_{1}$ and $x_{2}$ are weakly identified by $A$. Then, just as above, we have $Q_{1}$ (resp. $Q_{2}$ ) biholomorphic on $V_{1}$ (resp. $V_{2}$ ) and, as our spaces are locally irreducible, we may assume that $W_{1}=W_{2}$. Define $\varphi: V_{1} \rightarrow V_{2}$ by $\varphi=Q_{2}^{-1} \circ Q_{1}$ and $h: \mathscr{O}_{x_{2}} \rightarrow \mathcal{O}_{x_{1}}$ by $h(f)=f \circ \varphi$. Since $\varphi$ is biholomorphic it follows that $h$ is a continuous algebra isomorphism. It remains to show that $h$ extends $h_{x_{1}} \circ h_{x_{2}}^{-1}$. 
Let $f \in A$ be given and define $f_{i}$ by $f_{i}=\left.f\right|_{V_{i}}$. Then $\left(Q_{i}, f_{i}\right): V_{i} \rightarrow W_{i}^{\prime}$ is biholomorphic. Since $x_{1}$ and $x_{2}$ are assumed to be weakly identified by $A$, we may assume $W_{1}^{\prime}=W_{2}^{\prime}$. Now $W_{i}^{\prime}$ is the graph of $f_{i} \circ Q_{i}^{-1}$ on $W_{i}$ and $W_{1}=W_{2}$. Therefore, $f_{1} \circ Q_{1}^{-1}=f_{2} \circ Q_{2}^{-1}$. Hence $f_{2} \circ \varnothing=f_{1}$ or equivalently $h\left(f_{2}\right)=f_{1}$. Thus $h$ extends $h_{x_{1}} \circ h_{x_{2}}^{-1}$.

3. Construction of $\operatorname{Rep} A$. Our main goal is to construct envelopes of holomorphy for algebras of holomorphic functions, but in this section we consider abstract algebras over $C$ and construct their representative spaces.

DEFINITION 3.1. Let, $\mathcal{O}$ be the reduced structure sheaf for the normal complex space $V$ and ${ }_{V} \mathcal{O}_{p}$ its stalk at $p \in V$. Let $A$ be an algebra over $C$. A representation of $A$ on $V$ is an algebra homomorphism $\psi: A \rightarrow H(V)$. A local representation is an algebra homomorphism $\sigma: A \rightarrow{ }_{V} \mathcal{O}_{p}$ for some $p \in V$ such that $\sigma=h_{p} \circ \psi$, where $\psi$ is a representation of $A$ on $V$.

Let $\sigma: A \rightarrow{ }_{V} \mathcal{O}_{p}$ and $\rho: A \rightarrow{ }_{W} \mathcal{O}_{q}$ be local representations. We say that $\sigma$ and $\rho$ are equivalent if there are neighborhoods of $p$ and $q$ respectively, $V^{\prime}$ and $W^{\prime}$, in $V$ and $W$ and a biholomorphic map $\varphi: V^{\prime} \rightarrow W^{\prime}$ such that $\varphi(p)=q$ and $\varphi^{*}(\rho)=\sigma$. It is easy to check that equivalence of local representations is an equivalence relation. If $\sigma$ is a local representation of $A$, we will use [ $\sigma]$ to denote its equivalence class and if $\sigma$ and $\rho$ are equivalent local representations then we will denote that by $\sigma \sim \rho$.

The space $\operatorname{Rep} A$ will be composed of equivalence classes of local representations of $A$, each of which satisfies certain conditions.

Definition 3.2. Let $\sigma: A \rightarrow{ }_{V} \mathcal{O}_{p}$ be a local representation of $A$. We say that $\sigma$ is primitive if $\sigma=h_{p} \circ \psi$ such that

(1) The algebra $\psi[A]$ covers a variety at $p$ and

(2) There are quotients $q_{1}, \cdots, q_{m} \in \psi[A]^{*}$ which are holomorphic near $p$ on $V$ and give local coordinates at $p$ for $V$.

It is easy to check that if $\sigma$ is primitive and $\sigma \sim \rho$ then $\rho$ is also primitive. Thus we define an equivalence class $[\sigma]$ to be primitive if and only if $\sigma$ is primitive.

Definition 3.3. Let $A$ be an algebra over $C$. We define the representation space of $A, \operatorname{Rep} A$, as the collection of all primitive equivalence classes of local representations of $A$. 
We now proceed toward showing that $\operatorname{Rep} A$ can be given, in a natural way, the structure of a reduced, normal, not necessarily connected complex space. First, we cover Rep $A$ with a system of coordinate patches which gives it a topology. Consider $[\sigma] \in \operatorname{Rep} A$. Then $\sigma: A \rightarrow{ }_{V} O_{p}$ factors by

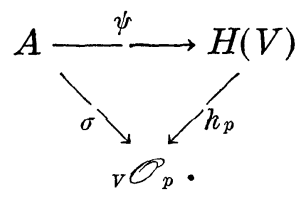

By taking $V$ smaller if necessary, we may assume that $\left[h_{x} \circ \psi\right]$ is primitive for every $x \in V$. Thus we have a map $i: V \rightarrow \operatorname{Rep} A$ defined by $i(x)=\left[h_{x} \circ \psi\right]$. Since $\psi[A]^{*}$ contains local coordinates at $p$ for $V$, we may take $V$ small enough for these to be coordinates for all of $V$. Thus $i$ is $1-1$. We give $i[V]$ the topological structure of $V$. Since $V$ has the complex structure of a normal, reduced complex space and since the change of coordinates on overlapping patches is biholomorphic, $\operatorname{Rep} A$ has induced complex structure. In order to show that $\operatorname{Rep} A$ has the structure of a normal, reduced, not necessarily connected complex space it is enough to show that our topology is Hausdorff.

Lemma 3.4. The topology defined above for $\operatorname{Rep} A$ is a Hausdorff topology.

Proof. Let $x_{1}, x_{2} \in \operatorname{Rep} A$. We suppose that any two coordinate neighborhoods of $x_{1}$ and $x_{2}$ have a nonempty intersection. In terms of representations, we have connected subvarieties $V_{1}$ and $V_{2}$ of open sets in complex Euclidean space with $x_{i} \in V_{i}$ and representations $\psi_{i}$ of $A$ on $V_{i}$ such that $\left[h_{x} \circ \psi\right]$ is primitive for $x$ in $V_{1}$ or $V_{2}$. Furthermore, there are sequences $\left\{x_{n}^{i}\right\} \subset V_{i}$ converging to $x_{i}$ such that

$$
\left[h_{x_{n}^{1}} \circ \psi_{1}\right]=\left[h_{x_{n}^{2}} \circ \psi_{2}\right] \text {. }
$$

In order to show that the topology is Hausdorff, we must show that $\left[h_{x_{1}} \circ \psi_{1}\right]=\left[h_{x_{2}} \circ \psi_{2}\right]$.

Let

$$
U_{1}=\left\{x \in V_{1} \mid \exists y \in V_{2} \text { with }\left[h_{x} \circ \psi_{1}\right]=\left[h_{y} \circ \psi_{2}\right]\right\}
$$

and

$$
U_{2}=\left\{y \in V_{2} \mid \exists x \in V_{1} \text { with }\left[h_{x} \circ \psi_{1}\right]=\left[h_{y} \circ \psi_{2}\right]\right\} .
$$

For every $x \in U_{1}$ there exists a $y \in U_{2}$, an open neighborhood $U_{x}\left(\operatorname{resp} . U_{y}\right)$ of $x$ (resp. $y$ ) and a biholomorphic map $\varphi_{x}: U_{x} \rightarrow U_{y}$ such that $\psi_{1}(f)=$ 
$\varphi_{x}^{*}\left(\psi_{2}(f)\right)$ on $U_{x}$ for every $f \in A$. In particular $U_{x} \subset U_{1}$ (resp. $U_{y} \subset U_{2}$ ) and thus $U_{1}$ (resp. $U_{2}$ ) is open in $V_{1}$ (resp. $V_{2}$ ). Furthermore, we claim that the $\operatorname{map} \varphi=\mathrm{U}_{x \in U_{1}} \varphi_{x}$ is well-defined on $U_{1}$ and a biholomorphic map of $U_{1}$ onto $U_{2}$.

To show that $\varphi$ is well-defined, consider $x \in U_{x_{1}} \cap U_{x_{2}}$. Suppose that $\varphi_{x_{1}}(x)=y_{1}$ and $\varphi_{x_{2}}(x)=y_{2}$. We have taken $V_{2}$ small enough so that there are quotients $q_{1}, \cdots, q_{n} \in \psi_{2}[A]^{*}$ which are holomorphic on $V_{2}$ and give coordinates there. Thus, if $y_{1} \neq y_{2}$ there is a $q_{i}$ (say $q_{1}$ ) such that $q_{1}\left(y_{1}\right) \neq q_{1}\left(y_{2}\right)$. But $q_{1}=\psi_{2}(f) / \psi_{2}(g)$, where $\psi_{1}(f)=\varphi_{x_{i}}^{*}\left(\psi_{2}(f)\right)$ for $i=1,2$ and similarly for $g$. Thus

$$
q_{1}\left(y_{2}\right)=\frac{\varphi_{x_{2}}^{*}\left(\psi_{2}(f)\right)}{\varphi_{x_{2}}^{*}\left(\psi_{2}(g)\right)}(x)=\frac{\psi_{1}(f)}{\psi_{1}(g)}(x)=\frac{\varphi_{x_{1}}^{*}\left(\psi_{2}(f)\right)}{\varphi_{x_{1}}^{*}\left(\psi_{2}(g)\right)}(x)=q_{1}\left(y_{1}\right)
$$

Hence $y_{1}=y_{2}$ and $\varphi$ is well-defined.

To show that $\varphi$ is a biholomorphic map of $U_{1}$ onto $U_{2}$, it is enough to show that it is injective. Suppose that $\varphi(a)=\varphi(b)=y$. If $a \neq b$ then there is a quotient $q \in \psi_{1}[A]^{*}$ such that $q(a) \neq q(b)$. If $q=\psi_{1}(f) / \psi_{1}(g)$ then by a similar argument to the above we find that $q(a)=q(b)$. Thus $a=b$ and $\varphi$ is $1-1$.

To summarize, we have a biholomorphic map $\varphi: U_{1} \rightarrow U_{2}$ such that for every $f \in A \varphi^{*} \psi_{2}(f)=\psi_{1}(f)$ on $U_{1}$. Further, we have assumed that $x_{i} \in \bar{U}_{i}$ for $i=1,2$. We will show that $x_{1} \in U_{1}$ and $x_{2} \in U_{2}$, thereby proving that $\left[h_{x_{1}} \circ \psi_{1}\right]=\left[h_{x_{2}} \circ \psi_{2}\right]$ and $\operatorname{Rep} A$ is Hausdorff.

Our first step is to show that $U_{2}$ is dense in $V_{2}$. By the primitivity assumption, there are functions $f_{1}, \cdots, f_{n} \in A$ such that $F_{1}=$ $\left(\psi_{1}\left(f_{1}\right), \cdots, \psi_{1}\left(f_{n}\right)\right)$ of $V_{1}$ onto a complex subvariety of an open set in $\boldsymbol{C}^{n}$, such that $\operatorname{dim} W=\operatorname{dim} V_{1}$. Let $F_{2}=\left(\psi_{2}\left(f_{1}\right), \cdots, \psi_{2}\left(f_{n}\right)\right)$. Now $\varphi$ is biholomorphic, $F_{1}$ is of generic maximal rank and $\varphi^{*} F_{2}=F_{1}$ on $U_{1}$. Thus $F_{2}$ is of generic maximal rank on $V_{2}$. Furthermore, $F_{2}^{-1}[W]$ must be a subvariety of $V_{2}$ which contains $x_{2}$. Let $S_{i}$ be the singular points of $V_{i}$ and $J_{i}$ be the points of $V_{i}-S_{i}$ where the Jacobian of $F_{i}$ vanishes, $i=1,2$. Let $U_{1}^{\prime}=U_{1}-S_{1}-J_{1}$ and $U_{2}^{\prime}=U_{2}-\varphi\left(S_{1}\right)-$ $\varphi\left(J_{1}\right)$. Observe that $x_{2} \in \bar{U}_{2}^{\prime}$. Now $F_{1}$ is locally $1-1$ on $U_{1}^{\prime}$ and therefore $F_{2}$ is locally $1-1$ on $U_{2}^{\prime}$. Furthermore, $U_{2}^{\prime} \subset F_{2}^{-1}[W]$. But $U_{2}^{\prime}$ being open in $V_{2}$ and $x_{2} \in \bar{U}_{2}^{\prime}$ implies that $\operatorname{dim}_{x_{2}} F_{2}^{-1}[W]=\operatorname{dim}_{x_{2}} V_{2}$. Thus, by taking $V_{2}$ smaller if necessary, we may assume that $F_{2}^{-1}[W]=V_{2}$.

Let $u_{2} \in \bar{U}_{2}$ such that

(1) $V_{2}$ is nonsingular at $u_{2}$,

(2) The Jacobian of $F_{2}$ is nonzero at $u_{2}$

and

(3) $u_{2} \notin F_{2}^{-1}\left[F_{1}\left[S_{1} \cup J_{1}\right]\right]$. 
By (1) and (2) there is a neighborhood $\Omega$ of $u_{2}$ contained in the nonsingular points of $V_{2}$ such that $\left.F_{2}\right|_{2}$ is a biholomorphic map. Let $u_{1} \in V_{1}$ be any point such that $F_{1}\left(u_{1}\right)=F_{2}\left(u_{2}\right)$. Since $F_{1}\left[V_{1}\right]=W \supset$ $F_{2}\left[V_{2}\right]$, we are assured that such points do exist. By (3) and by taking $\Omega$ smaller if necessary, $F_{1}^{-1} F_{2}$ exists and maps $\Omega$ biholomorphically onto an open neighborhood of $u_{1}$. Now $\Omega \cap U_{2} \neq \varnothing$ and

$$
\left.F_{1}^{-1} F_{2}\right|_{\varrho \cap U_{2}}=\left.\varphi^{-1}\right|_{\ell \cap U_{2}} \text {. }
$$

By uniqueness of analytic continuation $\left(F_{1}^{-1} F_{2}\right)^{*}$ has the same properties as $\left(\varphi^{-1}\right)^{*}$. Since $U_{2}$ is maximal for $\varphi^{-1}, \Omega \subseteq U_{2}$.

Let $B$ be the set of points in $V_{2}$ excluded by conditions (1)-(3) above. We will show that the Hausdorff dimension [4] of $B, H \operatorname{dim} B$, is at most $H \operatorname{dim} V_{2}-2$. Since $S_{2}$ and $J_{2}$ are analytic varieties of dimension at most $\operatorname{dim} V_{2}-1$, it is easily verified that their Hausdorff dimension is at most $H \operatorname{dim} V_{2}-2$. Thus, in order to show that $H \operatorname{dim} B \leqq H \operatorname{dim} V_{2}-2$, it is enough to show that $F_{2}^{-1}\left[F_{1}\left[S_{1} \cup J_{1}\right]\right]-$ $S_{2}-J_{2}$ has Hausdorff dimension at most $H \operatorname{dim} V_{2}-2$.

Now there is a collection of open subsets of $V_{2},\left\{A_{\nu}: \nu=1,2, \cdots\right\}$, such that $V_{2}-J_{2}-S_{2}=\bigcup_{\nu=1}^{\infty} A_{\nu}$ and $\left.F_{2}\right|_{A_{\nu}}$ is biholomorphic. Let $\mu_{\alpha}$ be the $\left(H \operatorname{dim} V_{2}-2+\alpha\right)$-dimensional Hausdorff measure. Hence

$$
\begin{gathered}
\mu_{\alpha}\left[F_{2}^{-1}\left[F_{1}\left[S_{1} \cup J_{1}\right]\right]-S_{2}-J_{2}\right] \leqq \sum_{\nu=1}^{\infty} \mu_{\alpha}\left[F_{2}^{-1}\left[F_{1}\left[S_{1} \cup J_{1}\right]\right] \cap A_{\nu}\right] \\
<<\sum_{\nu=1}^{\infty} \mu_{\alpha}\left[F_{1}\left[S_{1} \cup J_{1}\right] \cap F_{2}\left[A_{\nu}\right]\right] \leqq \sum_{\nu=1}^{\infty} \mu_{\alpha}\left[F_{1}\left[S_{1} \cup J_{1}\right]\right]
\end{gathered}
$$

where the symbol $<<$ means that the left hand side is zero if the right hand side is zero. Since $\operatorname{dim}\left(S_{1} \cup J_{1}\right) \leqq \operatorname{dim} V_{2}-1, F\left[S_{1} \cup J_{1}\right]$ is a countable union of complex varieties (almost thin) each of which has dimension at most $\operatorname{dim} V_{2}-1$. If $\alpha>0$ then, by the countable subadditivity of $\mu_{\alpha}, \mu_{\alpha}\left[F_{1}\left[S_{1} \cup J_{1}\right]\right]=0$. Therefore, the above inequalities show that, for $\alpha>0, \mu_{\alpha}\left[F_{2}^{-1}\left[F_{1}\left[S_{1} \cup J_{1}\right]\right]-S_{2}-J_{2}\right]=0$. As a result $H \operatorname{dim} B \leqq H \operatorname{dim} V_{2}-2$.

We have previously shown that $\partial U_{2} \subseteq B$. Thus $H \operatorname{dim} \partial U_{2} \leqq$ $H \operatorname{dim} V_{2}-2$. Now, $U_{2}$ and $V_{2}-\bar{U}_{2}$ are disjoint open sets separated by a set of Hausdorff codimension at least 2. This is impossible [4] unless one of the two sets is empty. Therefore, $V_{2}-\bar{U}_{2}=\varnothing$ and $U_{2}$ is dense in $V_{2}$.

We have shown that $V_{1}$ and $V_{2}$ can be chosen such that $\varphi: U_{1} \rightarrow U_{2}$ is biholomorphic onto $U_{2}$, which is an open dense subset of $V_{2}$. Suppose $\psi_{1}(f) / \psi_{1}(g) \in \psi_{1}[A]^{*}$ is holomorphic on $V_{1}^{\prime} \supset \supset V_{1}$. Now

$$
\psi_{2}(f) / \psi_{2}(g) \circ \varphi=\psi_{1}(f) / \psi_{1}(g)
$$

on $U_{1}$. Thus $\psi_{2}(f) / \psi_{2}(g)$ is bounded on the dense set $U_{2}$ and, since 
$V_{2}$ is normal, is therefore holomorphic at $x_{2}$.

We could have performed the same proof for $U_{1}$ to show that $\varphi: U_{1} \rightarrow U_{2}$ is biholomorphic from the open set $U_{1}$, which is dense in $V_{1}$. Thus, using the analogous argument to the above, $\psi_{2}(f) / \psi_{2}(g)$ holomorphic on $V_{2}^{\prime} \supset \supset V_{2}$ implies $\psi_{1}(f) / \psi_{1}(g)$ holomorphic at $x_{1}$. We thus conclude that $\psi_{1}(f) / \psi_{1}(g)$ is holomorphic at $x_{1}$ if and only if $\psi_{2}(f) / \psi_{2}(g)$ is holomorphic at $x_{2}$.

By primitivity, there are functions $f_{1}^{i}, \cdots, f_{n_{i}}^{i}$ and $g_{1}^{i}, \cdots, g_{n_{i}}^{i}$ in $A$ such that the quotients $\psi_{i}\left(f_{1}^{i}\right) / \psi_{i}\left(g_{1}^{i}\right), \cdots, \psi_{i}\left(f_{n_{i}}^{i}\right) / \psi_{i}\left(g_{n_{i}}^{i}\right)$ are holomorphic on $V_{i}$ and give coordinates there for $i=1,2$. Define

$$
G_{i}: V_{i} \rightarrow C^{n_{1}+n_{2}}
$$

by

$$
\begin{aligned}
G_{i}= & \left(\psi_{i}\left(f_{1}^{1}\right) / \psi_{i}\left(g_{1}^{1}\right), \cdots, \psi_{i}\left(f_{n_{1}}^{1}\right) / \Psi_{i}\left(g_{n_{1}}^{1}\right),\right. \\
& \left.\psi_{i}\left(f_{1}^{2}\right) / \psi_{i}\left(g_{1}^{2}\right), \cdots, \psi_{i}\left(f_{n_{2}}^{2}\right) / \psi_{i}\left(g_{n_{2}}^{2}\right)\right)
\end{aligned}
$$

for $i=1,2$. Then $G_{i}$ is biholomorphic on $V_{i}$ and $G_{2} \circ \varphi=G_{1}$ on $U_{1}$.

Now $G_{i}\left[V_{i}\right]$ is an irreducible normal subvariety containing $G_{1}\left(x_{1}\right)=$ $G_{2}\left(x_{2}\right)$. Furthermore,

$$
G_{1}\left[V_{1}\right] \cap G_{2}\left[V_{2}\right] \supseteqq G_{1}\left[U_{1}\right]=G_{2}\left[U_{2}\right]
$$

and $G_{i}\left(x_{i}\right) \in \overline{G_{i}\left[U_{i}\right]}$ for $i=1,2$. Therefore,

$$
\operatorname{dim}_{G_{1}\left(x_{1}\right)} G_{1}\left[V_{1}\right]=\operatorname{dim}_{G_{1}\left(x_{1}\right)} G_{1}\left[V_{1}\right] \cap G_{2}\left[V_{2}\right]=\operatorname{dim}_{G_{2}\left(x_{2}\right)} G_{2}\left[V_{2}\right] .
$$

Hence, by choosing $V_{1}$ and $V_{2}$ appropriately, we may assume that $G_{1}\left[V_{1}\right]=G_{2}\left[V_{2}\right]$. Thus $G_{2}^{-1} \circ G_{1}$ is a biholomorphic extension of $\varphi$ to a map from $V_{1}$ to $V_{2}$. Furthermore, by the unicity of analytic continuation, this has the property $\left(G_{2}^{-1} \circ G_{1}\right)^{*} \psi_{2}(f)=\psi_{1}(f)$ for every $f \in A$. Thus $U_{1}=V_{1}$, if $V_{1}$ is chosen small enough, and $\left[h_{x_{1}} \circ \psi_{1}\right]=$ $\left[h_{x_{2}} \circ \psi_{2}\right]$.

COROLlary 3.5. Endowed with the topology described above, Rep $A$ is a reduced, normal, not necessarily connected complex space.

4. The main theorems. In order to prove Theorem 2 and Theorem 3, we need several preliminary lemmas.

Lemma 4.1. Let $V$ be a complex space and $A \subseteq H(V)$ an algebra which has generic maximal rank on $V$. Suppose there are holomorphic maps $\varphi_{i}: V \rightarrow V_{i}$ onto the complex spaces $V_{i}$ and algebras $A_{i} \subseteq H\left(V_{i}\right)$ such that $\varphi_{i}^{*}: A_{i} \rightarrow A$ is an algebra isomorphism. Suppose further that for some $p \in V\left[h_{\varphi_{1}(p)} \circ\left(\varphi_{1}^{*}\right)^{-1}\right]$ and $\left[h_{\varphi_{2}(p)} \circ\left(\varphi_{2}^{*}\right)^{-1}\right]$ are primitive. Then $\left[h_{\varphi_{1}(p)} \circ\left(\varphi_{1}^{*}\right)^{-1}\right]=\left[h_{\varphi_{2}(p)} \circ\left(\varphi_{2}^{*}\right)^{-1}\right]$. 
Proof. The maps $\varphi_{i}$ have generic maximal rank on $V$. Let $S$ be the singular points of $V$ and $J_{i}$ be the subset of $V-S$ where the Jacobian of $\varphi_{i}$ vanishes. Clearly $U=V-S-J_{1}-J_{2}$ is an open dense subset of $V$. The map $\varphi_{2} \circ \varphi_{1}^{-1}$ is locally defined and locally biholomorphic on $\varphi_{1}[U]$. Now, the isomorphism of $A$ and $A^{i}$ yields the existence of a unique $f^{i} \in A^{i}$ such that $f=f^{i} \circ \varphi_{i}$ for every $f \in A$. Thus, locally on $\varphi_{1}[U], f^{1}=f^{2} \circ\left(\varphi_{2} \circ \varphi_{1}^{-1}\right)$. Therefore, since $U$ is dense, we can obtain a sequence $\left\{x_{n}\right\}$ in $U$ which converges to $p$ such that $\varphi_{i}\left(x_{n}\right) \rightarrow \varphi_{i}(p)$ and $\left[h_{\varphi_{1}\left(x_{n}\right)} \circ\left(\varphi_{1}^{*}\right)^{-1}\right]=\left[h_{\varphi_{2}\left(x_{n}\right)} \circ\left(\varphi_{2}^{*}\right)^{-1}\right]$. For $n$ sufficiently large, these equivalence classes of local representations must be primitive. But Rep $A$ is Hausdorff. Thus $\left[h_{\varphi_{1}(p)} \circ\left(\varphi_{1}^{*}\right)^{-1}\right]=\left[h_{\varphi_{2}(p)} \circ\left(\varphi_{2}^{*}\right)^{-1}\right]$.

Let $A$ be an algebra over $C$ and $\operatorname{Rep} A$ its representative space. For $[\sigma] \in \operatorname{Rep} A$, define $\hat{f}([\sigma])=\sigma(f)(p)$, where $\psi: A \rightarrow H(V)$ and $\sigma=$ $h_{p} \circ \psi$. Clearly the definition of $\hat{f}$ is independent of the particular representative of the equivalence class. If $V$ is taken sufficiently small it is biholomorphically equivalent to a coordinate neighborhood of $[\sigma]$ in $\operatorname{Rep} A$ and $\hat{f}$ is defined by $\psi(f)$ on $V$. Thus $\hat{f}$ is holomorphic on $\operatorname{Rep} A$. We define the algebra homomorphism $\hat{h}: A \rightarrow \hat{A}$ by $\hat{h}(f)=\hat{f}$.

Lemma 4.2. The algebra $\hat{A}$ weakly separates points on $\operatorname{Rep} A$ and $\hat{A}^{*}$ gives local coordinates at each $p \in \operatorname{Rep} A$.

Proof. It follows immediately from the definition of primitivity that $\hat{A}^{*}$ gives local coordinates at each $p \in \operatorname{Rep} A$.

Let $x_{1}, x_{2} \in \operatorname{Rep} A$. Suppose that every $\hat{q} \in \hat{A}^{*}$ which is holomorphic at $x_{1}$ is holomorphic at $x_{2}$ and vice versa. Also assume that for such holomorphic quotients, $\hat{q}\left(x_{1}\right)=\hat{q}\left(x_{2}\right)$. Under these hypotheses, we will show that $\operatorname{dim}_{\left(x_{1}, x_{2}\right)} S_{\hat{A}} \geqq \operatorname{dim} X$ implies that $x_{1}=x_{2}$.

Let $V_{1}$ and $V_{2}$ be coordinate neighborhoods of $x_{1}$ and $x_{2}$ respectively. Since $x_{i}$ is a primitive representation of $A$, there are quotients $\widehat{q}_{1}^{i}, \cdots, \hat{q}_{n_{i}}^{i}$ holomorphic on $V_{i}$ such that $Q_{i}=\left(\widehat{q}_{1}^{i}, \cdots, \hat{q}_{n_{i}}^{i}\right)$ is biholomorphic on $V_{i}$. Now, by our assumption, $Q_{1}$ is holomorphic on $V_{2}$ and $Q_{2}$ is holomorphic on $V_{1}$. Therefore, $\left(Q_{1}, Q_{2}\right)$ is biholomorphic on both $V_{1}$ and $V_{2}$ such that $\left(Q_{1}, Q_{2}\right)\left(x_{1}\right)=\left(Q_{1}, Q_{2}\right)\left(x_{2}\right)$. Let $G_{i}=\left.\left(Q_{1}, Q_{2}\right)\right|_{V_{i}}$. Since $\operatorname{dim}_{\left(x_{1}, x_{2}\right)} S_{\hat{A}} \geqq \operatorname{dim} X$, we may assume, by taking $V_{i}$ smaller if necessary, that $G_{1}\left[V_{1}\right]=G_{2}\left[V_{2}\right]$. Define $\varphi: V_{1} \rightarrow V_{2}$ by $\phi=G_{2}^{-1} \circ G_{1}$, for every $f \in A$, let $\hat{f}_{i}=\left.\hat{f}\right|_{V_{i}}$. For the same reasons as above,

$$
\left(G_{1}, \hat{f}_{1}\right)\left[V_{1}\right]=\left(G_{2}, \hat{f}_{2}\right)\left[V_{2}\right] \text {. }
$$

Thus $\hat{f}_{2} \circ \varphi=\hat{f}_{1}$ for every $f \in A$. Therefore, since $\varphi\left(x_{1}\right)=x_{2}, x_{1}=x_{2}$. Recall that an algebra $A$ of functions holomorphic on a complex 
space $X$ is ample on $X$ if for every $p \in X A$ covers a variety at $p$ and there is a neighborhood $W$ of $p$, a holomorphic mapping $\varphi$ of $W$ onto a complex space $V$ and an algebra $\hat{A} \subset H(V)$ such that $\varphi^{*}: \hat{A} \rightarrow A$ is an algebra isomorphism and $\hat{A}^{*}$ contains local coordinates at each point of $V$.

LEMMA 4.3. Let $A$ be an ample algebra of functions holomorphic on a complex space $X$. Then there is a unique holomorphic map $\tau: X \rightarrow \operatorname{Rep} A$ such that $\tau^{*}: \hat{A} \rightarrow A$ is an algebra isomorphism with $\tau^{*-1}=\hat{h}$. The set $\tau[X]$ is an open subset of $\operatorname{Rep} A$ and the map $\tau$ is 1-1 if and only if $A$ weakly separates points on $X$.

Proof. Since $A$ is ample on $X$, we have for each $p \in X$ the map $\varphi$ in the definition of ampleness. We define $\tau$ by $\tau(p)=\left[h_{\varphi(p)} \circ\left(\varphi^{*}\right)^{-1}\right]$. The map $\tau$ is clearly holomorphic and $\tau^{*-1}(f)=\hat{f}$. By Lemma 4.1, $\tau$ is unique. The $\varphi$ guaranteed by the ampleness of $A$ maps onto a complex space $W$. Thus a biholomorphic copy of $W$ is contained in $\tau[W]$ with $\tau(p)$ corresponding to $\varphi(p)$. Therefore, $\tau[X]$ is open. It remains to show that $\tau$ is $1-1$ if and only if $A$ weakly separates points on $X$.

Let $x_{1}, x_{2} \in X$ and suppose $\tau\left(x_{1}\right)=\tau\left(x_{2}\right)$. Thus there are neighborhoods $V_{i}$ of $x_{i}$ and a biholomorphic map $\varphi: V_{1} \rightarrow V_{2}$ such that $f_{1}=$ $f_{2} \circ \varphi$, where $f_{i}=\left.f\right|_{V_{i}}$, for every $f \in A$. In particular, both conditions for the weak identification of $x_{1}$ and $x_{2}$ by $A$ are satisfied.

Conversely, suppose that $x_{1}$ and $x_{2}$ are weakly identified by $A$. Let $V_{i}$ be a neighborhood of $x_{i}$ so that on $W_{i}=\tau\left[V_{i}\right]$ the map $\hat{Q}_{i}=$ $\left(\widehat{q}_{1}^{i}, \cdots, \widehat{q}_{n_{i}}^{i}\right)$ is biholomorphic, where $\hat{q}_{j}^{i} \in \hat{A}^{*}$ and $i=1,2$. Since $A$ weakly identifies $x_{1}$ and $x_{2}, q_{j}^{i}$ is holomorphic at both $x_{1}$ and $x_{2}$ and $q_{j}^{i}\left(x_{1}\right)=q_{j}^{i}\left(x_{2}\right)$ for every $i$ and $j$. Let $\widehat{G}_{i}=\left.\left(\hat{Q}_{1}, \hat{Q}_{2}\right)\right|_{W_{i}}$. Clearly $\hat{G}_{i}$ is biholomorphic on $W_{i}$. Now $G_{i}=\hat{G}_{i} \circ \tau$ maps $V_{i}$ onto $\hat{G}_{i}\left[W_{i}\right]$ and, since $\operatorname{dim}_{\left(x_{1}, x_{2}\right)} S_{A} \geqq \operatorname{dim} X$, we can choose $V_{i}$ appropriately so that $G_{1}\left[V_{1}\right]=G_{2}\left[V_{2}\right]$. Thus $\hat{G}_{1}\left[W_{1}\right]=\widehat{G}_{2}\left[W_{2}\right]$ and we have a biholomorphic $\operatorname{map} \varphi: W_{1} \rightarrow W_{2}$ defined by $\varphi=\widehat{G}_{2}^{-1} \circ \widehat{G}_{1}$. For exactly the same reasons as in the proof of Lemma $4.2, \hat{f}_{1}=\hat{f}_{2} \circ \varnothing$, where $\hat{f}_{i}=\left.\hat{f}\right|_{W_{i}}$, for every $\hat{f} \in \hat{A}$. Since $\varphi\left(\tau\left(x_{1}\right)\right)=\tau\left(x_{2}\right), \tau\left(x_{1}\right)=\tau\left(x_{2}\right)$.

We now proceed with the main theorems.

THeorem 2. Let $A$ be an algebra over $C$. Then there is a not necessarily connected) complex space $\operatorname{Rep} A$, and an algebra $\hat{A} \subseteq H(\operatorname{Rep} A)$ such that each component of $\operatorname{Rep} A$ is weakly Stein with respect to $\hat{A}$. Furthermore, there is an algebra homomorphism $\hat{h}$ of $A$ onto $\hat{A}$ such that for any other algebra homomorphism $h^{\prime}$ of $A$ onto $A^{\prime}$, where $A^{\prime}$ is an ample algebra on a complex space $X^{\prime}$, there is a unique holo- 
morphic map $\varphi$ of $X^{\prime}$ onto an open subset of $\operatorname{Rep} A$ such that for every $f \in A, h^{\prime}(f)=\hat{h}(f) \circ \varnothing$. The map $\varnothing$ is 1-1 if and only if $A^{\prime}$ weakly separates points on $X^{\prime}$.

Proof. By the previous lemmas, in order to prove each component of $\operatorname{Rep} A$ is weakly Stein with respect to $\hat{A}$, it is enough to verify the maximality condition, $(\delta)$ in Definition 1.3. Thus we let $X$ be a component of $\operatorname{Rep} A$ and suppose $X$ is an open subset of a complex space $X^{\prime}$. Suppose $A^{\prime}$ is an algebra of functions holomorphic on $X^{\prime}$ such that the restriction map $r: A^{\prime} \rightarrow A$ is an algebra isomorphism. Finally we assume that $A^{\prime}$ and $X^{\prime}$ satisfy conditions $(\alpha),(\beta)$, and $(\gamma)$ in the definition of weakly Stein. Since $r$ is an isomorphism, $\operatorname{Rep} A=\operatorname{Rep} A^{\prime}$. Now $A^{\prime}$ weakly separates points on $X^{\prime}$. Thus Lemma 4.3 gives us a $1-1$ holomorphic mapping $\tau: X^{\prime} \rightarrow \operatorname{Rep} A$. Since $\tau$ is unique it must agree with the identity on $X$. But $\tau\left[X^{\prime}\right]$ is connected and therefore $\tau\left[X^{\prime}\right] \subset X$. Hence $\tau\left[X^{\prime}\right]=X$ and $X^{\prime}=X$.

The algebra homomorphism $\hat{h}$ has already been constructed. Now $h^{\prime}: A \rightarrow A^{\prime}$ is a homomorphism onto an ample algebra on $X^{\prime}$. Thus, for every primitive local representation $\left[\sigma^{\prime}\right]$ of $A^{\prime}$, we obtain a primitive local representation $\left[\sigma^{\prime} \circ h^{\prime}\right]$ of $A$. In this way we obtain a biholomorphic injection $i: \operatorname{Rep} A^{\prime} \rightarrow \operatorname{Rep} A$ onto an open subset of $\operatorname{Rep} A$. Now Lemma 4.3 gives us the map $\tau^{\prime}: X^{\prime} \rightarrow \operatorname{Rep} A^{\prime}$ such that $\tau\left(x_{1}^{\prime}\right)=\tau\left(x_{2}^{\prime}\right)$ if and only if $x_{1}^{\prime}$ and $x_{2}^{\prime}$ are weakly identified by $A^{\prime}$. Let $\varphi=i \circ \tau$. The uniqueness of $\phi$ follows from Lemma 4.1. Clearly $\varphi$ is 1-1 if and only if $A^{\prime}$ weakly separates points on $X^{\prime}$. It remains to check that $h^{\prime}(f)=\hat{h}(f) \circ \varnothing$ for every $f \in A$, but this follows by a simple diagram chase.

THEOREM 3. Let $A$ be an ample algebra of functions holomorphic on a complex space $X$. Then there is a complex space $E$ and an algebra $\hat{A} \subset H(E)$ such that

(1) The space $E$ is weakly Stein with respect to $\hat{A}$.

(2) There is a holomorphic mapping $\tau$ of $X$ onto an open subset of $E$ such that $\tau^{*}: \hat{A} \rightarrow A$ is an algebra isomorphism.

(3) If $\tau^{\prime}$ is a holomorphic mapping of $X$ onto an open subset of a complex space $X^{\prime}$ and there is an ample algebra $A^{\prime} \subset H\left(X^{\prime}\right)$ such that $\tau^{\prime *}: A^{\prime} \rightarrow A$ is an isomorphism then there is a unique holomorphic mapping $\Phi$ of $X^{\prime}$ onto an open subset of $E$ such that for every $f \in A$, $\left(\tau^{*}\right)^{-1}(f) \circ \varphi=\left(\tau^{*}\right)^{-1}(f)$. The map $\varphi$ is 1-1 if and only if $A^{\prime}$ is weakly separating on $X^{\prime}$.

Proof. The map $\tau$ is given by Theorem 2 by letting $X=X^{\prime}$ and $E$ is taken to be the connected component of $\operatorname{Rep} A$ which contains $\tau[X]$. Then $E$ is weakly Stein with respect to $\hat{A}$. Now $\tau^{*-1}=\hat{h}$ 
must be an isomorphism, because $\tau[X]$ is an open subset of $E$.

The map needed for (3) is also given by Theorem 2 and the unicity of $\tau$ implies that $\tau=\varphi \circ \tau^{\prime}$ on $X$. Thus $\varphi\left[X^{\prime}\right] \subset E$ and $\left(\tau^{*}\right)^{-1}(f) \circ \varphi=\left(\tau^{*}\right)^{-1}(f)$.

5. Concluding remarks. It should be observed that the entire question of the existence of $\tau: X \rightarrow \operatorname{Rep} A$ has been avoided by building it into the definition of ampleness. This is a drawback of our theory, but the study of existence seems to be quite complicated. At this point in time we strongly believe that the existence question should be asked in the category of meromorphic maps instead of holomorphic maps.

Even though the existence question has been avoided here, our theory applies in many classical cases. For example suppose $A=H(X)$, where $X$ is a reduced, irreducible normal complex space. Further, assume that $H(X)$ separates points and gives local coordinates. Then our theory applies and we obtain the weak envelope of holomorphy for the pair $(X, H(X))$. In particular, this applies to the type of space $X$ and algebra $H(X)$ constructed by Grauert [1], where $H(X)$ is not a Stein algebra.

\section{REFERENCES}

1. H. Grauert, Bemerkenswerte pseudoconvexe Mannigfaltigkeiten, Math. Z., 87 (1963).

2. R. C. Gunning and H. Rossi, Analytic Functions of Several Complex Variables, Prentice Hall, 1965.

3. A. T. Huckleberry, Holomorphic Mappings and Algebras of Holomorphic Functions of Several Complex Variables, Stanford University Dissertation, 1970.

4. W. Hurewicz and H. Wallman, Dimension Theory, Princeton, 1941.

5. H. Royden, Algebras of bounded analytic functions on Riemann surfaces, Acta Math., 114 (1965).

6. K. Stein, Maximale holomorphe und meromorphe Abbildungen, II, Amer. J. of Math., 86 (1964).

Received March 30, 1972. Partially supported by N.S.F. grant GP 20139.

UNIVERSITY OF NOTRE DAME 


\section{PACIFIC JOURNAL OF MATHEMATICS}

\section{EDITORS}

D. Gilbarg and J. Milgram

Stanford University

Stanford, California 94305

R. A. Beaumont

University of Washington

Seattle, Washington 98105
J. DUGUNDJI* Department of Mathematics

University of Southern California Los Angeles, California 90007

RICHARD ARENS

University of California

Los Angeles, California 90024

\section{ASSOCIATE EDITORS}

E. F. BeCKenbach

B. H. NeUMaNN

F. WOLF

K. Yoshida

\section{SUPPORTING INSTITUTIONS}

UNIVERSITY OF BRITISH COLUMBIA

UNIVERSITY OF SOUTHERN CALIFORNIA

CALIFORNIA INSTITUTE OF TECHNOLOGY

UNIVERSITY OF CALIFORNIA

MONTANA STATE UNIVERSITY

STANFORD UNIVERSITY

UNIVERSITY OF TOKYO

UNIVERSITY OF NEVADA

UNIVERSITY OF UTAH

NEW MEXICO STATE UNIVERSITY

WASHINGTON STATE UNIVERSITY

OREGON STATE UNIVERSITY

UNIVERSITY OF OREGON

OSAKA UNIVERSITY

UNIVERSITY OF WASHINGTON

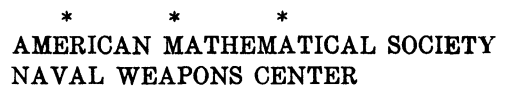

* C. DePrima will replace J. Dugundji until August 1974. 


\section{Pacific Journal of Mathematics}

\section{Vol. 47, No. $1 \quad$ January, 1973}

K. Adachi, Masuo Suzuki and M. Yoshida, Continuation of holomorphic

mappings, with values in a complex Lie group ....................

Michael Aschbacher, A characterization of the unitary and symplectic groups

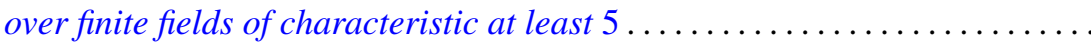

Larry Eugene Bobisud and James Calvert, Energy bounds and virial theorems for abstract wave equations....................................

Christer Borell, A note on an inequality for rearrangements ................

Peter Southcott Bullen and S. N. Mukhopadhyay, Peano derivatives and general

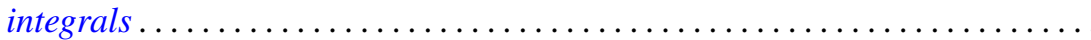

Wendell Dan Curtis, Yu-Lee Lee and Forrest Miller, A class of infinite dimensional subgroups of $\operatorname{Diff}^{r}(X)$ which are Banach Lie groups .........

Paul C. Eklof, The structure of ultraproducts of abelian groups ...............

William Alan Feldman, Axioms of countability and the algebra $C(X) \ldots \ldots \ldots$

Jack Tilden Goodykoontz, Jr., Aposyndetic properties of hyperspaces...........

George Grätzer and J. Płonka, On the number of polynomials of an idempotent algebra. II ...........................................

Alan Trinler Huckleberry, The weak envelope of holomorphy for algebras of

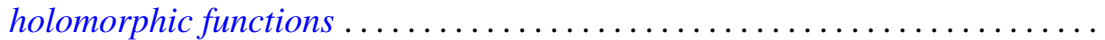

John Joseph Hutchinson and Julius Martin Zelmanowitz, Subdirect sum decompositions of endomorphism rings . . . . . . . . . . . . . . . .

Gary Douglas Jones, An asymptotic property of solutions of

$y^{\prime \prime \prime}+p y^{\prime}+q y=0$.

Howard E. Lacey, On the classification of Lindenstrauss spaces .

Charles Dwight Lahr, Approximate identities for convolution measure algebras.

George William Luna, Subdifferentials of convex functions on Banach

spaces.

Nelson Groh Markley, Locally circular minimal sets. .

Robert Wilmer Miller, Endomorphism rings of finitely generated projective modules

Donald Steven Passman, On the semisimplicity of group rings of linear

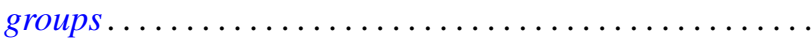

Bennie Jake Pearson, Dendritic compactifications of certain dendritic spaces.

Ryōtarō Satō, Abel-ergodic theorems for subsequences ...... .

Henry S. Sharp, Jr., Locally complete graphs. . .

Harris Samuel Shultz, A very weak topology for the Mikusinski field of

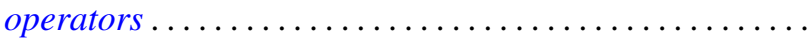

Elena Stroescu, Isometric dilations of contractions on Banach spaces ...

Charles W. Trigg, Versum sequences in the binary system ... . .

William L. Voxman, On the countable union of cellular decompositions of n-manifolds 